This item was submitted to Loughborough's Research Repository by the author.

Items in Figshare are protected by copyright, with all rights reserved, unless otherwise indicated.

\title{
The effects of turbulence on jet stability and the flame transfer function in a lean-burn combustor
}

PLEASE CITE THE PUBLISHED VERSION

https://doi.org/10.1080/00102202.2020.1777992

PUBLISHER

Informa UK Limited

VERSION

AM (Accepted Manuscript)

\section{PUBLISHER STATEMENT}

This is an Accepted Manuscript of an article published by Taylor \& Francis in Combustion Science and Technology on 18 Jul 20, available online: https://doi.org/10.1080/00102202.2020.1777992

\section{LICENCE}

CC BY-NC-ND 4.0

\section{REPOSITORY RECORD}

Treleaven, Nick, Andrew Garmory, and Gary Page. 2020. "The Effects of Turbulence on Jet Stability and the Flame Transfer Function in a Lean-burn Combustor”. Loughborough University.

https://hdl.handle.net/2134/12727115.v1. 


\title{
SUBMISSION TO: COMBUSTION SCIENCE AND TECHNOLOGY
}

\section{The effects of turbulence on jet stability and the flame transfer function in a lean-burn combustor}

\author{
N. C. W. Treleaven ${ }^{\mathrm{a}}$, A. Garmory ${ }^{\mathrm{b}}$ and G. J. Page ${ }^{\mathrm{b}}$ \\ aSTFS, TU-Darmstadt, Darmstadt, Germany; b Loughborough University, Loughborough, \\ United Kingdom
}

ARTICLE HISTORY

Compiled June 2, 2020

\begin{abstract}
Large eddy simulations show that the penetration of the central jet in a multipassage lean burn and liquid fuelled combustor is dependent on the turbulence levels in the three air-flow passages of the injector. These simulations are performed using an incompressible method where an unsteady boundary condition is applied to the inlets of a truncated domain which only includes the domain downstream of the fuel injector using the recently developed Proper Orthogonal Decomposition Fourier Series method. The fluctuating inlets are built from a combination of compressible URANS data and incompressible LES data. This incompressible method is shown to be consistent with fully compressible simulations whilst requiring only one third of the computing time. Neglecting the turbulence generated in the passages results in the incorrect penetration of the central jet, resulting in a flame transfer function with a similar gain but with a different phase. Furthermore, large scale helical modes, previously detected in non-reacting simulations of a similar burner geometry are seen to be imprinted onto the liquid fuel spray, mixture fraction and heat release fields. This shows that coupling between hydrodynamic instabilities and thermoacoustic instabilities in liquid fuelled engines may be more significant than suggested by previous studies of gas fuelled engines.
\end{abstract}

\section{KEYWORDS}

Combustion; PODFS; Thermoacoustics;

\section{Nomenclature}

' fluctuating component

12 variance

* non-dimensionalised quantity

- mean component

$\Delta \quad$ mesh cell size

$\dot{q} \quad$ heat release rate

$\kappa \quad$ wavenumber

$\mathcal{F} \quad$ flame transfer function

$\rho \quad$ density

CONTACT N. C. W. Treleaven. Email: treleaven@stfs.tu-darmstadt.de 


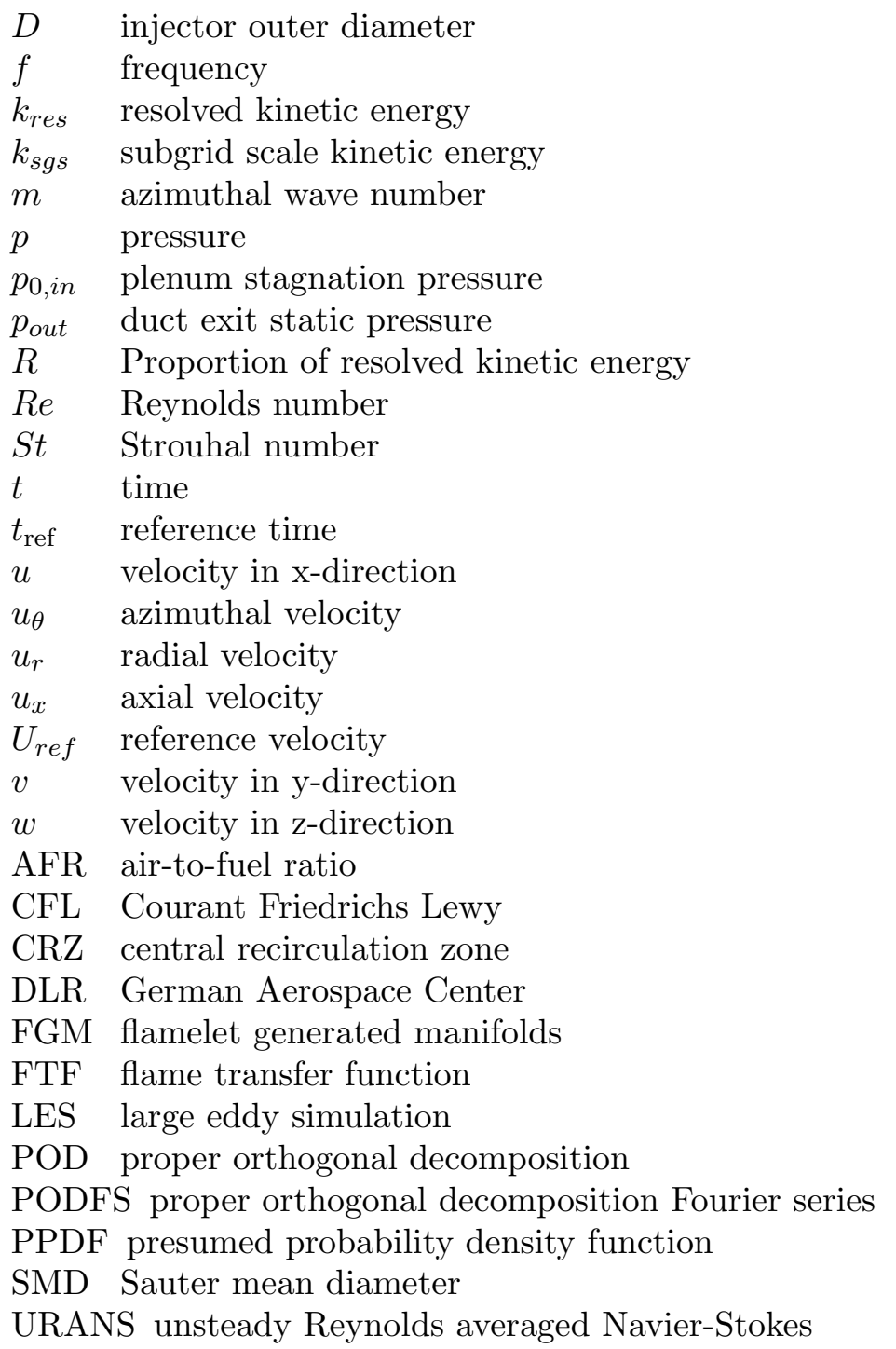

\section{Introduction}

Lean-burn combustion systems for gas turbines are especially susceptible to combustion instabilities. These instabilities are the result of a feedback mechanism between the heat release of the flame, the acoustics and the flow field inside the combustion chamber. A popular strategy for predicting instabilities involves the splitting of the phenomenon into processes related to the acoustic field and processes related to the flow field and flame (Noiray, Durox, Schuller, and Candel (2008)). The acoustic field may be resolved using a number of non-linear (Caraeni, Devaki, Aroni, Oswald, and Caraeni (2009)), linearised (Nicoud, Benoit, Sensiau, and Poinsot (2007)) or semianalytical acoustic network models (Dowling and Stow (2003)) which require as their input a flame transfer function (FTF) which links heat release fluctuations of the flame with velocity, pressure, or mass flow rate fluctuations at a reference point inside the combustion chamber. The FTF can be derived using experimental methods or using large eddy simulation (LES) (see for instance: Candel, Durox, Schuller, Bourgouin, and Moeck (2014), Chong, Komarek, Kaess, Foller, and Polifke (2010), Palies, Schuller, 
Durox, Gicquel, and Candel (2011), Dupuy et al. (2020) and Merk et al. (2019)). For compressible simulations or experiments, the flame is usually acoustically forced from the upstream or downstream boundary while the mass flow rate or velocity and heat release rate is monitored. These experiments and compressible LES simulations are relatively expensive and make it difficult to compute the FTF of a new burner design early in the design process where the designer may iterate the design several times to achieve the desired emissions, flame stability or other favourable characteristics.

In studies on swirl stabilised premixed flames, Palies, Schuller, Durox, and Candel (2011) showed that the response of the flame could be accurately captured by considering the interaction of the flame with a) axial velocity fluctuations, which lead to flame-vortex interaction, and b) fluctuations of azimuthal velocity that lead to changes in the swirl number and the turbulent flame speed. More recently Dupuy et al. (2020) have shown that models based on these mechanisms and mean flow parameters can be used to closely represent an experimentally derived flame transfer function. Other studies have suggested further mechanisms; Thumuluru and Lieuwen (2009) suggested that the flame response is driven by a) annular jet fluctuations, b) oscillatory turbulent flame brush development, c) flame stabilisation and d) fluid mechanical instabilities. Although all of these things can interact with or are caused by the incoming acoustic waves, they do not involve direct interaction between the flame and the acoustic wave but rather through one or more intermediary processes.

It was shown by Treleaven, Su, Garmory, and Page (2019) that the magnitude of axial velocity fluctuation at the exit of the fuel injector caused by an incoming acoustic wave is several times higher in magnitude than the acoustic particle velocity experienced in the region of the flame. This high magnitude velocity fluctuation in the injector exit is convected to the flame by the mean flow field and does not propagate at the speed of the sound. This implies that the response of the flame should be able to be captured using an incompressible methodology provided that the acoustically forced, fluctuating flow field downstream of the injector is captured accurately by the method. This assumption may suffer inaccuracies at very low forcing frequencies due to direct interactions between the flame and the acoustic waves, however at these low frequencies the flame transfer function approaches the value of $1+0 \imath$ as demonstrated by Polifke and Lawn (2007).

It was also shown by Treleaven, $\mathrm{Su}$, et al. (2019) that the downstream fluctuations of axial velocity and swirl number could be accurately captured using the proper orthogonal decomposition Fourier series (PODFS) method where the acoustic response of the fuel injector is first investigated using non-reacting compressible unsteady Reynolds averaged Navier-Stokes (URANS) simulations. In these simulations the fluctuating velocity of the air at the exit of the fuel injector passages is recorded over a small number of acoustic cycles. This information can then be applied to the inlets of a reacting incompressible LES simulation on a truncated domain that only includes the combustor geometry downstream of the injector flow passage outlets. The application of this unsteady flow field data to the inlets of the truncated domain is facilitated through use of the PODFS method.

However, whilst application of the inlet fields generated using URANS data includes sufficient information on the interaction of the acoustic waves with the fuel injector, it contains no fluctuating velocity contribution from the turbulence generated in the injector flow passages. Neglecting the second of these contributions results in the incorrect penetration of the central jet, the so called "flipped" configuration as seen by Treleaven, Garmory, and Page (2019), and found in a similar configuration in the experimental study of Williams, Carrotte, Moran, and Walker (2018) and the numerical 
study of Langella et al. (2020). The same effect can be emulated by reducing the mass flow rate in the central jet. The flipped configuration is characterised by the centrally located pilot flame opening up into a "V" shape as seen in Figure 1. It has been shown in Treleaven, Staufer, Spencer, Garmory, and Page (2020) that the penetration of the central jet can be corrected using turbulence generated by the digital filter method of Klein, Sadiki, and Janicka (2003)

As described by Williams et al. (2018), the relative mass flow rates through the different combustor passages may be altered by installation effects, such as the location of upstream air feeds, the compressor pre-diffuser, or the design of the fuel injector. All of these inputs will also contribute to the generation of turbulence inside the fuel injector passages, which may be dynamically altered by the presence of an acoustic instability (see Kirthy, Hemchandra, Hong, Shanbhogue, and Ghoniem (2016) and Treleaven, Juniper, Su, Garmory, and Page (2018)). This gives the designer of such a fuel injector an additional parameter that may be modified to adjust the response of the flame to acoustic perturbations.

Helical modes, as first observed by Lambourne (1962), are large coherent flow structures common in swirling flows. They are caused by Kelvin-Helmholtz hydrodynamic instabilities that originate in regions of high shear as described in Oberleithner et al. (2011) and Juniper (2012) among others. It has been shown by Treleaven, Su, Garmory, and Page (2017), using a similar fuel injector and in a non-reacting case, that the downstream flow field contains helical modes of azimuthal wave number $m=1$ and $m=2$ whilst acoustic forcing induces axisymmetric $m=0$ modes. Furthermore it is seen that the helical and acoustic modes can interact, modifying the strength of the helical modes depending on the level and frequency of forcing (see Kirthy et al. (2016), Terhaar, Ćosić, Paschereit, and Oberleithner (2016) and Treleaven et al. (2018)). In the numerical study of Treleaven, Garmory, and Page (2020), it was shown that there is a high correlation between helical modes and the dynamics of the fuel spray while in the numerical and experimental study of Keller et al. (2015), the droplets are also seen to be influenced by the precessing vortex core (PVC), an $m=1$ helical mode. It was shown in Treleaven, Garmory, and Page (2019) that localised fluctuations of the fuel spray diameter can lead to large changes in the heat release rate. Whilst in the experimental study of Renaud, Ducruix, and Zimmer (2019), the higher momentum of the fuel droplets allows these helical modes to penetrate further into the flow field than the velocity fluctuations that proceed them. This means that hydrodynamically generated flow structures may contribute to localised fluctuations of heat release more significantly in liquid fuelled combustion chambers than previously seen in lab-scale, gas-fuelled burners such as in the study of Steinberg, Boxx, Stöhr, Meier, and Carter (2012). In this paper, the interaction of helical modes with the fuel spray and heat release fields is analysed using POD (Proper Orthogonal Decomposition).

This study shows how variations of upstream turbulence can significantly affect the downstream flow field and hence, the FTF of an industrial swirl burner. It is also shown that the cost of simulations to resolve the FTF can be significantly reduced by considering the hybrid PODFS approach with similar results as compared to the conventional fully compressible approach. The downstream flow field is also seen to induce large scale flow structures that are also shown to interact strongly with the fuel spray, mixture fraction fields and the local heat release rate. 


\section{Methodology}

\subsection{Computational Domain}

The injector used in this study is a liquid fuelled lean-burn injector designed for application in large civil aircraft jet engines. It comprises of three air flow swirler passages (A, B and C) and two liquid fuel atomisers. This is the same injector as investigated by Treleaven, Garmory, and Page (2019) and is visualised in Figure 2. A centrally located pilot flame is fuelled by a pressure swirl atomiser located at the downstream tip of a bullet located in the centre of passage A (purple cross in Figure 3 ) while air is provided to the flame by flow passage $\mathrm{A}$. The main flame forms a ring around the pilot flame where air is provided to the flame by passages $\mathrm{B}$ and $\mathrm{C}$ while fuel is atomised by an air-blast atomiser located between these two passages (green crosses in Figure 3). The air is swirled in the opposite direction in passage A as compared to passages $\mathrm{B}$ and $\mathrm{C}$ to improve mixing.

The computational domain is the SCARLET rig (SCaled ACoustic Rig for Low Emmision Technology) located at DLR in Cologne. The rig consists of an axissymetric single sector burner set up for lean-burn aero-engine combustion tests surrounded by two long cylindrical ducts. At each end of the ducts sit four acoustic sirens (two upstream and two downstream) which are capable of acoustically exciting the flow from either upstream or downstream at high amplitude. Further on from each of the sirens lie an upstream and downstream damper designed to prevent sound waves leaving the rig from being reflected back towards the combustor. The rig inlet flow can be heated up to $950 \mathrm{~K}$ and the whole rig can also be pressurised up to 30 Bar. Along each duct lies an array of five microphones that can be used to monitor the pressure waves travelling along the ducts which then allows the measurement of the FTF through the multi-microphone and Rankine Hugenot relations as described in Polifke (2015). The rig also allows for the measurement of heat release rates by monitoring the $O H^{*}$ chemiluminescence.

The workflow used in this study is shown in Figure 4: Firstly, non-reacting compressible URANS simulations are used to obtain the acoustic response of the fuel injector as described in Section 2.2. Then in Section 2.3 an incompressible reacting LES simulation is run to obtain the FTF of the burner through use of the PODFS method to apply the acoustic response of the fuel injector to the incompressible domain. In this simulation it was observed that the pilot jet flow from passage A was in the flipped configuration. It was hypothesised that this was due to insufficient turbulence being fed into the combustion chamber due to the PODFS models being generated from URANS data. To solve this problem an auxiliary non-reacting and incompressible LES was run to obtain the turbulent fields at the passage exit planes as described in Section 2.4. In Section 2.5, this additional turbulent information was captured using additional PODFS models and applied to a further incompressible reacting simulation along with the original acoustic PODFS models in a similar way to Section 2.3. In this secondary case, the pilot jet was observed to be penetrating into the domain and the FTF was also calculated for this flow configuration. The auxiliary non-reacting URANS simulations use different computational domains and meshes to the LES simulations of the flame. In Section 2.6 the sensitivity of the results are tested by computing the same reacting case on a lower resolution mesh. Finally in Section 2.7 the results are compared to a reacting compressible case at two different frequencies of forcing to test the validity of the incompressible methodology. 


\subsection{Obtaining the acoustic response of the fuel injector}

The mean and acoustically modulated velocity field at these three inlet planes is provided by an auxiliary simulation of the full SCARLET rig including the full injector geometry following the methodology of Su, Garmory, and Carrotte (2015) and Treleaven et al. (2017). This auxiliary simulation is a compressible URANS simulation on a relatively coarse mesh ( 5 million cells) that captures the interaction of the fuel injector with acoustic waves propagating upstream from the downstream acoustic sirens. This mesh is shown in Figures 3 and 5. The magnitude of the acoustic wave was set to $p^{\prime} / \bar{p}=0.003$ following the work of Treleaven et al. (2017) and the frequency set to $S t=0.14$ which corresponds to a frequency of instability seen in a real engine geometry. The Strouhal number is defined by:

$$
S t=\frac{f D}{U_{\text {ref }}}=f t_{\mathrm{ref}}
$$

where $D$ is the injector diameter and hence $t_{\text {ref }}$ is:

$$
t_{\mathrm{ref}}=\frac{D}{U_{\text {ref }}}
$$

and the reference velocity is defined as:

$$
U_{\text {ref }}=\sqrt{\frac{2\left(p_{0, \text { in }}-p_{\text {out }}\right)}{\rho}}
$$

where $p_{0, \text { in }}$ is the upstream total pressure, $p_{\text {out }}$ is the downstream static pressure and $\rho$ is the upstream air density. The operating conditions of the rig were chosen to be representative of an engine close to take-off thrust. This is consistent with a Reynolds number of $R e=1.2 \times 10^{6}$ and a Mach Number of $M=0.23$ based on the injector diameter and the reference velocity. $p_{0, \text { in }}-p_{\text {out }}$ was set to being equal to $0.04 p_{0, \text { in }}$ corresponding to the pressure drop required to achieve the same mass flow rate as seen in engine tests with the same injector geometry.

All other variables have been non-dimensionalised by the maximum spatial value of the temporal mean, for example, the heat release rate becomes:

$$
\dot{q}^{*}=\frac{\dot{q}}{\max (\overline{\dot{q}})}
$$

All simulations were run using PRECISE-UNS, an in-house CFD solver developed by Rolls-Royce. Further details are found in Anand, Eggels, Staufer, Zedda, and Zhu (2013). The momentum equations were solved using a second order TVD (Total Variation Diminishing) method and the pressure using a second order method. Temporal integration is provided by a second order backwards method. In the case of this first compressible auxiliary simulation, the enthalpy of the flow is also discretised using a first order upwind method, and together with the ideal gas equation, takes into account compressible effects in the flow. Turbulence closure was provided by the $k-\omega$ SST 
model as described by Menter, Kuntz, and Langtry (2003) with transport equations for $k$ and $\omega$ being discretised using a first order upwind method. The simulation was run for 4000 time steps of $2 \times 10^{-1} t_{\text {ref }}$ with no acoustic forcing to obtain a psuedosteady flow solution before then being run for a few thousand time steps to remove any transients and then for 12 acoustic cycles with a time step of $2 \times 10^{-3} t_{\text {ref }}$ with acoustic waves injected through the downstream boundary using the non-reflective characteristic boundary conditions described in Treleaven et al. (2017).

After the transients had passed and during this acoustically forced compressible simulation, the velocity was interpolated every $50^{\text {th }}$ time step onto three $200 \times 200$ point planes whose normals are aligned in the positive $\mathrm{x}$-direction and axially located such that those are in line with the exit of each of the three flow passages of the fuel injector (see Figure 6). In order to facilitate the application of this plane data onto the inlets of the truncated domain, the PODFS method was used for each flow passage as described in Treleaven, $\mathrm{Su}$, et al. (2019) with the Fourier series energy set to 0.9 and using the first 20 POD modes. Contained within these 3 PODFS models is the temporal mean flow and the fluctuations of velocity through the fuel injector as caused by the imposed acoustic waves.

\subsection{The incompressible simulation without turbulent fluctuations imposed at the inlet}

In order to obtain the FTF of this injector the computational domain was truncated to only include the rig geometry downstream of the three flow passage exits of the fuel injector. These three injector exits then become the inlets to the truncated simulation geometry. This was previously done in Treleaven, Garmory, and Page (2019) and this study uses the same computational mesh. This truncated domain was run assuming incompressible flow with the three PODFS models obtained from the auxiliary URANS simulation providing the fluctuating velocity field to the three inlets that represent the outlets of the fuel injector. The simulation was first run for 4000 iterations with only the mean components of the velocity field imposed at the inlets with a time step of $2 \times 10^{-1} t_{\text {ref }}$ to obtain a pseudo-steady solution. Dodecane fuel was injected as Lagrangian parcels from the first time step at 100 equidistant points around the circumference of the main flame atomiser and at 50 points around the circumference of the pilot atomiser. The fuel parcel diameters were chosen at random according to a Rosin-Rammler distribution with a mean SMD (Sauter mean diameter) of $2.2 \times 10^{-4} D$. The evaporation of the fuel droplets is assumed to follow the work of Lefebvre and Chin (1983). The flow was ignited after 100 iterations by heating a small portion of the flow to a high enough temperature to initialise the reaction.

Combustion modelling followed the FGM-PPDF (flamelet generated manifolds presumed probability function) approach as described further in Treleaven, Garmory, and Page (2019) and Anand et al. (2013). A transport equation is solved for enthalpy, mixture fraction, an unscaled progress variable and the variances of the mixture fraction and the progress variable. These five control variables are then linked to the state of the flame through an FGM table generated using CHEM1D as described in Hermanns (2001) assuming premixed flamelets with the dodecane reaction mechanism of Nehse, Warnat, and Chevalier (1996). Turbulence-flame interaction is taken into account through pre-integration of the FGM output variables assuming a beta-PDF for the relationship of the progress variable and its variance and a 3-delta-PDF for the relationship of mixture fraction and its variance (see Robin, Mura, Champion, and 
Plion (2006)). Temperature is interpolated from the FGM table and local enthalpy calculation using the method of Vicquelin, Fiorina, Payet, Darabiha, and Gicquel (2011).

As in the compressible auxiliary simulation, velocity and pressure are solved using a second order method in space while all other variables were solved using an upwind method. Temporal integration was also conducted using a backwards second order method and turbulence closure provided by the $k-\omega$ SST model. After the 4000 initialisation time steps, the PODFS models were then turned on, the turbulence model replaced by the Smagorinsky subgrid scale model (Smagorinsky (1963)) and the time step reduced to $2 \times 10^{-3} t_{\text {ref. }}$ The simulation was then run for 40,000 time steps to allow the turbulent field to develop and the FTF to be calculated by monitoring the mass flow rate through the inlets and the integral heat release rate fluctuations and computing (see Vold, Crowley, and Rocklin (1984)):

$$
\mathcal{F}(\omega)=\frac{S_{\dot{m} \dot{Q}}}{S_{\dot{m} \dot{m}}}
$$

where $S_{\dot{m} \dot{Q}}$ is the cross-spectrum of mass flow and heat release rates and $S_{\dot{m} \dot{m}}$ is the power spectral density of the mass flow rate. During the initial stages of the calculation, the jet coming from passage A was seen to open up into the "flipped" configuration as previously described. It was hypothesised that it might be caused by insufficient small scale turbulent fluctuations being imposed at the inlets.

\subsection{The auxiliary incompressible LES}

The PODFS models built using the auxiliary compressible URANS calculation contain no information about how turbulence develops in the injector flow passages and propagates downstream into the combustion chamber. In order to take this turbulence into account, an additional non-reacting incompressible unforced LES calculation was run of the similar fuel injector geometry presented in Treleaven, Su, et al. (2019), using the same numerical methodology and with a 3\% pressure drop between upstream plenum total pressure and downstream static pressure. In this simulation, the flow was recorded at the exit planes of the injector passages using the same $200 \times 200$ point plane mesh and three additional PODFS models, one for each flow passage, were constructed from the results. In this case the simulation was run for 100,000 time steps of $1 \times 10^{-3} t_{\text {ref }}$ with the PODFS models built from the last 30,000 time steps using every $200^{t h}$ time step. The computational domain contained 69 million cells and was checked agains the criterion described in Pope (2001), using the method described in Dianat, McGuirk, Fokeer, and Spencer (2014) and Treleaven, Garmory, and Page (2019). The first 30 modes were used with a Fourier energy coefficient of 0.9. These three new PODFS models (with mean flow removed) were then added to the original three PODFS models to create three 50 mode PODFS models for each injector inlet. These new models then contain information about both the acoustic forcing and turbulence generated in the flow passages.

\subsection{The incompressible simulation with turbulent fluctuations imposed at the inlet}

The same simulation as described in Section 2.3 was then repeated using this second set of PODFS models that contain information about both the acoustic and turbulent 
fluctuations at the inlet. In addition to turbulence being included at the inlet, the mean inlet mass flow through Passage A was temporarily boosted to 1.5 times the mass flow rate specified by the PODFS model by multiplying the mean inlet velocity field by a factor of 1.5 to help the jet stabilise during the 4000 time steps of flow initialisation. After this 4000 time steps the mass flow rates of the three inlets were adjusted to the same values as seen in the compressible auxiliary simulation. This was achieved by boosting the mean mass flow rates of passages $\mathrm{A}, \mathrm{B}$ and $\mathrm{C}$ by $1.20,1.12$ and 1.01 respectively. This adjustment of the mean mass flow rates by scaling of the inlet velocities is required due to interpolation errors that occur during the transfer of data between the original compressible simulation data, the PODFS mesh and the incompressible mesh inlet. Additional error is also due to the the changes in the density field between the compressible non-reacting simulation and the incompressible reacting density field.

This strategy resulted in a strongly penetrating and stable pilot flame jet, this flow configuration will be referred to as "coring" in the remainder of this paper. The simulation was again run for 40,000 steps as in the flipped flow configuration case. To ensure that this flow configuration was stabilised by the introduction of turbulence and not due to the change of mass flow rates, the simulation was run again from a stable coring jet flow initial solution with increased mass flow rates imposed at the inlets with the non-turbulent PODFS models. In this case, the pilot jet was seen to switch back to the flipped configuration after a few thousand time steps. This shows that the turbulent fluctuations generated in the flow passaged are essential to stabilise the pilot jet. The FTF for this coring jet configuration was also calculated using the same method as in Section 2.3. This same methodology was then repeated with acoustic forcing at $S t=0.30$.

\subsection{The effects of mesh resolution}

In order to test the sensitivity of this LES methodology to mesh density, the same simulation as in Section 2.5 was run on a coarser computational mesh. In this case, the mesh was coarsened in the region of the flame to $0.014 D$, twice the cell size of the high resolution case. This low resolution incompressible mesh contains around six million cells.

\subsection{The effects of compressibility}

The use of the incompressible PODFS methodology is dependent on the fluctuations of heat release being driven by convective processes and not due to direct interaction between the acoustic waves and the flame. In order to test this hypothesis, two compressible reacting simulations were run to compare against the PODFS methodology. These simulations were run at forcing frequencies of $S t=0.14$ and $S t=0.3$.

In order to reproduce the results from the experiment, the simulation domain includes the SCARLET geometry from just downstream of the upstream sirens to just upstream of the downstream sirens. The meter panel that separates the upstream duct from the outer annulus of the combustion chamber is included in the computational domain however the cooling features are replaced with effusion boundary conditions that simply remove mass from one surface and inject it at another.

Density boxes are used to control the mesh size in critical areas: The injector flow passages are limited to $0.007 D$ while the area around the meter panel and the area 
downstream of the injector is limited to $0.014 D$, the same resolution as the low resolution incompressible case. The global cell size was limited to $0.08 D$. The mesh, excluding the ducts, was generated using the octree mesh generation algorithm of ICEMCFD, with several iterations of smoothing and mesh coarsening. Following this the mesh was converted to a mixed hexahedral-tetrahedral type and the ducts created by extruding the mesh with a constant cell size of $0.08 \mathrm{D}$. This resulted in a mesh of around 15 million cells. The mesh densities used were based on the work of Treleaven et al. (2017) scaled by the relative magnitudes of the speed of sound between each simulation to ensure that the acoustic waves were accurately captured. The centre section of this mesh is shown in Figure 6.

The upstream and downstream boundary conditions were made to be non-reflective using the methods described in Treleaven et al. (2017) however the upstream boundary condition was relaxed towards the required mass flow to ensure the correct AFR was achieved in the combustor. Acoustic waves of magnitude $p^{\prime}=0.007 p_{0, \text { in }}$ were introduced from these boundaries, as required, also using the methods described in Treleaven et al. (2017). Data was collected for 100,000 timesteps once any transients were overcome. Due to the large size of the domain, it could take up to 100,000 additional steps to come to a statistically steady state. The numerical methodology in this case is the same as the other reacting cases except that the flow field is assumed to be compressible. This is achieved by calculating the density in each cell using the local pressure, temperature and specific gas constant. The only additional changes in numerical methodology between the incompressible and compressible simulations is the inclusion of a pressure source term in the enthalpy equation and the inclusion of the divergence of the velocity field in the calculation of the fluid stress tensor.

In order to compute the FTF for this incompressible case, the velocity and density of the fluid at the exit plane of the injector (see Figure 6) is interpolated onto a $200 \times 200$ cell mesh and the instantaneous mass flow rate calculated. The heat release rate is calculated in the same way as in the incompressible simulations.

\section{Results}

Figure 7 shows the time and azimuthally averaged flow field for the two incompressible reacting LES simulations. In the case of the coring flow configuration the central pilot jet coming from passage A can be seen to be penetrating into the centre of the domain and the central recirculation zone (CRZ) that is formed by the two other passage jets. In the case of the flipped configuration, the pilot jet is split open and is seen to wrap around the CRZ instead. The size of the CRZ is also increased in the flipped case. As shown in Figure 8, in the case of the coring flow, the central part of the flow in the combustion chamber is seen to rotate in the opposite direction to the flow located further radially outwards. In the case of the flipped configuration, the entire flow is seen to rotate in the same direction as the main jets with the exception of the flipped pilot jet.

In Figure 1 the mean and azimuthally averaged heat release fields are shown for the two flow configurations. In the case of coring flow, the pilot flame is seen to be in a detached state and forming a "W" shape in the centre of the domain whilst in the case of a flipped pilot jet, the pilot flame is much closer to the injector and forms a "V" shape. In both cases the pilot flame intensity is much higher than the main flame despite $80 \%$ of the fuel being fed to the main flame atomiser, this is due to the much smaller volume of the pilot flame that lies along the centreline of the 
cylindrical combustion chamber and the amount of air fed to the flame. The main flame is relatively similar across both cases except that the flame is seen to extend further upstream and be slightly more intense in the flipped case where is almost attaches to the outer edge of injector Passage C.

The FTFs of the two cases are shown in Figure 9. This shows that both simulations produce an FTF gain that quite similar to each other. The phase however shows that the two simulations produce an FTF with a totally different phase relationship.

Figure 10 shows the resolved proportion of kinetic energy in the LES simulation of the coring simulation and the turbulent spectra at select locations in the domain. The resolved proportion of kinetic energy is calculated using the method of Moeng and Wyngaard (1988):

$$
R=\frac{k_{\text {res }}}{k_{\text {res }}+k_{\text {sgs }}}
$$

where:

$$
k_{r e s}=\frac{1}{2}\left(u^{\prime 2}+v^{\prime 2}+w^{\prime 2}\right)
$$

and the sub-grid scale kinetic energy is approximated using:

$$
k_{s g s}=0.099 \Delta^{2}\left(2 \bar{S}_{i j} \bar{S}_{i j}\right)
$$

where $\bar{S}_{i j}$ is the symmetric part of the mean strain rate tensor:

$$
\bar{S}_{i j}=\frac{1}{2}\left(\frac{\partial \bar{u}_{i}}{\partial x_{j}}+\frac{\partial \bar{u}_{j}}{\partial x_{i}}\right)
$$

Figure 10a shows that whilst there are some regions of the flow that are under-resolved close to the walls and in the core of the central jet, the proportion of resolved kinetic energy is above $80 \%$ over most of the domain and in the regions of the flow where the flame is active. In addition, the turbulent spectra has been approximated using Taylor's hypothesis at the three points marked in Figure 10a. The first point is located along the centreline of the domain, the third point is located in the centre of the main flame jet coming from passages $\mathrm{B}$ and $\mathrm{C}$ and the second point is located midway between these points. The three points are located $0.1 D$ downstream of the injector exit plane. These three spectra show that the gradient of the spectral function with respect to the wavenumber in the mean flow direction is steeper than $-5 / 3$ as shown in the Figures 10b, 10c and 10d in orange and as predicted by Kolmogorov (1941).

\subsection{Compressibility and mesh resolution}

Figure 11 shows the gain and phase of the FTF calculated for the high resolution incompressible simulations for two frequencies as compared to a fully compressible simulation and a low resolution incompressible simulation. The low resolution incompressible simulation and the compressible simulation have the same mesh resolution. 
These results show that the FTF as calculated between the compressible and incompressible simulations have very similar phase, especially at the higher forcing frequency. There is some difference in the calculation of gain, with a higher gain being found in the high resolution incompressible simulations. The incompressible low resolution simulation shows a lower gain which suggests that discrepancy of gain between the high resolution incompressible simulations and compressible simulations is mostly due to the modified mesh density and not the numerical method. Table 1 shows that the hybrid PODFS method, whilst being more complex is overall significantly cheaper than the more traditional compressible methodology.

\section{2. $P O D$}

The proper orthogonal decomposition (POD) of the fluctuating flow variables inside the combustion chamber was computed for the coring reacting flow case forced at $S t=0.14$ to see whether helical modes are present in this reacting case and whether they interact with the fuel spray and heat release fields. The POD was computed using the snapshot POD method of Sirovich (1987) with the inner product defined by the fluctuating and normalised values of velocity, spray mixture fraction, spray momentum, progress variable, mixture fraction, heat release rate, temperature and pressure (see Treleaven, Garmory, and Page (2019) for further details). Similarly to the non-reacting compressible case presented in Treleaven et al. (2017), the flow was seen to contain helical modes with azimuthal wavenumber of $m=0, m=1$ and $m=2$. As also seen in the non-reacting two-phase study of Treleaven, Garmory, and Page (2020), these helical modes, along with a $m=3$ helical mode, could also be seen to interact with the fuel spray. Figure 12 shows the spatial component of the second highest energy containing POD mode for axial velocity, axial momentum of the fuel spray, mixture fraction and heat release rate.

The POD was also computed for the non-reacting compressible URANS auxiliary simulation and the incompressible LES auxiliary simulation. In these cases, as the flow is single phase and non-reacting, only the velocity was used in the calculation of the inner product. Table 2 shows the azimuthal wavenumber as calculated using the method described in Treleaven et al. (2018), for the 10 highest energy containing modes for the 2 auxillary and coring LES simulations.

\section{Discussion}

It is relatively well understood that the phase of the FTF can be related to the delay between when fluctuations of velocity at the inlet exit plane are produced and when those fluctuations interact with the flame (see for example Schuller, Durox, and Candel (2003)). The observation that in this case the FTF has shifted phase between the flipped and coring flow configurations can be seen as a direct consequence of the shift of the flame position inside the combustion chamber.

The flow fields in the region of the main flame between the coring and flipped cases are quite similar except that in the flipped case the pilot jet is also seen to interact more strongly with the other two main flame jets, increasing the dispersion of these jets further downstream. Despite the similarity of the flow field in the region of the main flame, the main flame in the flipped configuration is seen to be shifted upstream and strengthened by the flipped pilot jet. This extension upstream of the main flame in the reacting case is due to some of the fuel from the pilot atomiser being thrown 
outwards and into the main flame in this case. The pilot flame also feeds hot reaction products forward towards the injector and then outwards to the main flame. In the case of the flipped configuration the distance between two flames is reduced and hence the evaporation and reaction rate in the main flame in enhanced in this case, which causes the flame to move towards the injector.

In order to stabilise a thermoacoustically unstable combustion chamber fitted with this fuel injector it would be necessary to increase damping or modify the FTF. Modification of the FTF may include design modifications to reduce the gain or modifications to induce a shift of the phase such that the sensitivity of the burner is shifted away from resonance frequencies of the combustion chamber. It is clearly shown in these computations that the phase of the FTF can be drastically shifted through moving the flame in the combustion chamber. In this case, it is achieved through inducing a quite radical change in the mean flow field, however, it is also seen that the interaction between the two flames plays an important role in the location of the main flame. Adjusting the separation of the two flames would likely also induce a shift of the FTF phase. Furthermore, the observed large change in the mean flow field is induced through a relatively minor change in the boundary conditions. In the real engine case, the strength of turbulent fluctuations at the exit of the injector flow passages will be dependent on many factors including the output flow from the compressor, the relative location of the pre-diffuser and fuel injector, the design of the flow passages, and the design of the swirl vanes. All of these parameters can be altered to either induce a coring or flipped configuration. It may also be possible to design a system that when it becomes unstable, induces an alternative flow configuration that then stabilises the combustion system through an associated shift in the FTF phase. Given the strong link between the location of the flame and the phase of the FTF, it is interesting to consider whether the FTF can be approximated by considering the mean flow field only and this will be the focus of future work.

This analysis highlights the importance of turbulent fluctuations in determining the stability of the combustion system. Different levels of inlet turbulence will result in a different flame location and therefore a different phase of the FTF. This suggests that modifying the injector geometry to promote or dampen the generation of turbulence could be an effective strategy for stabilising an unstable system. Furthermore, the sensitivity of the flow to upstream turbulence means that for any simulation aimed at resolving the flame stability, the upstream boundary condition must include an accurate representation of the upstream turbulent fluctuations in a real engine.

\subsection{Compressibility and mesh resolution}

Figure 10 shows that the majority of the turbulent kinetic energy is resolved in the higher resolution incompressible simulations while the increased steepness of the spectra implies that the vast majority of the inertial subrange is well resolved by the LES mesh. The FTF results shown in Figure 11 show that the calculation of the FTF is quite insensitive to the numerical method and mesh resolution, especially the phase. The phase information is arguably more important than the gain as the true response of the system and the gain of the FTF is likely to be non-linear as described by Candel et al. (2014) among others. This suggests that during the preliminary design stage of the injection system, the mesh could be coarsened to reduce the cost associated with resolving the FTF to allow for rapid assessment of future designs.

The accuracy of the resulting FTF could potentially be improved by considering 
alternative numerical methods. The second order TVD scheme employed for the momentum fluxes and the first order scheme employed for the calculation of enthalpy are notoriously dissipative and may impact unfavourably upon the results. These schemes are required in the case of the compressible simulations as small scale features in the combustion chamber and fuel injector result in small cells that at the chosen time step results in relatively high CFL numbers. In industrial systems these small scale features are more prevalent than in lab-scale systems and as a result offer an additional challenge for numerical modelling. This highlights a further advantage of the incompressible PODFS method: Because the computational domain is truncated and much simpler, the quality of the computational mesh is much higher, leading to better solver stability. This enables higher order and less dissipative spatial schemes to be used however in this study it was important that the methodology was as consistent as possible between the incompressible and compressible simulations to ensure that errors were better representative of the true differences between compressible and incompressible numerical methods and not due to the method of resolving the numerical fluxes.

\section{2. $P O D$}

Table 2 shows that the strength of helical modes is dependent on the geometry, operating point and whether or not the flow is reacting. All three simulations show that the highest energy containing flow structures are $m=2$ helical modes, represented by two POD modes. In the case of the two LES calculations, the second highest energy containing flow structure is an $m=1$ helical mode while in the URANS simulation, it is an axisymmetric $m=0$ mode. In the case of the non-reacting LES simulation, the existence of the $m=1$ mode is due to the slightly different geometry and operating point from the other two simulations. The preference of the $m=0$ mode in the compressible non-reacting case is driven by the acoustic forcing, which is not present in the incompressible non-reacting LES. In the case of the reacting simulation, $m=0$ modes appear due to the acoustic forcing imposed at the inlet while the appearance of the $m=1$ mode is driven either by the change in density field due to the reaction and two phase flow, or as an artefact of the use of the PODFS model which contains remnants of the the $m=1$ mode from the incompressible LES.

The generation of helical modes occurs in the wave maker region, the overlap of the globally unstable mode and its adjoint as described in Chomaz (2005). Provided that the incompressible reacting LES simulation includes this region, the generation of helical modes will be accurately predicted and is not due to the imposed artefacts in the PODFS models. In Treleaven et al. (2018) it was attempted to obtain the global unstable modes and their adjoints for the $m=1$ and $m=2$ helical modes in a similar geometry to the one in this study using local linear stability analysis. Whilst the global mode and its adjoint were not successfully found in this study, a local, absolutely unstable $m=2$ mode was found in the region between the exits of passage A and B, just downstream of the exit of passage A. This region is very likely the wave maker region for this flow due to the high shear in the mean velocity which leads to the highest observed growth rate in the local stability analysis and therefore, the observed $m=1$ mode in the reacting simulation is generated from the flow and is not an artefact of the use of the PODFS models at the inlet.

In Figure 12 all four spatial components of the second highest energy POD mode for the coring reacting LES simulation show a clear $m=2$ signature. The strength of 
the mode for the axial velocity is shown to be larger, closer to the combustion chamber centreline than the other three mode components. As seen in the experimental study of Renaud et al. (2019), the reason the spray momentum, mixture fraction and heat release rate components of the mode penetrate further into the combustion chamber is because the fuel spray, once accelerated by the helical mode in the velocity field, has a much higher momentum than the surrounding fluid and hence requires more time to breakdown. This observation, along with observations made in Treleaven, Garmory, and Page (2019) that the interaction of the spray with the wall can strongly drive heat release fluctuations, suggests that in liquid fuelled combustion chambers, the effect of helical modes on the fluctuating heat release rate, may be much stronger than in gas fuelled combustors. Furthermore, while helical modes fluctuations of heat release rate cannot contribute to longitudinal combustion instabilities in symmetrical combustion chambers, they could become synchronised with instabilities that propagate around the annulus of a fully annular combustion system. If the distance from the wall to the fuel injector is different on the inner and outer annulus of such a combustion chamber, and interactions of the fuel spray with the wall could lead to fluctuations of heat release, then the helical mode may also contribute to longitudinal thermoacoustic instabilities in real engines. In a real engine combustion chamber the fuel injector would be located in a ring shaped annulus along with several other injectors around the ring. The injector location could be biased towards either the outer or inner wall of such a combustion chamber. As the fuel mass fraction fluctuations spiral outwards from the centre of each injector, the axial location at which the spiral hits the outer wall will be different to the inner wall. If the heat release rate is closely correlated to the time when this helical structure interacts with each wall then the axial location of maximum heat release will fluctuate at the frequency of the rotation of this helical structure, allowing for a net contribution to a longitudinal thermo-acoustic instability.

\section{Conclusion}

In this study two incompressible reacting LES simulations have been run to investigate the effect of inlet turbulence on the flow field and FTF of a lean-burn injector geometry. The simulations were acoustically forced through use of a series of PODFS models. In the first simulation, the PODFS models were built from a non-reacting compressible auxiliary simulation that captured the interaction of the acoustic wave with the swirler but neglected the turbulent velocity fluctuations generated in the passages of the fuel injector. This resulted in the incorrect penetration of the central pilot jet and a downstream "flipped" configuration of the flow. An additional auxiliary LES simulation was run to generate realistic turbulent fluctuations at the exit planes of the injector passages and these fluctuations were also modelled using the PODFS method.

A second reacting LES simulation was run with velocity fluctuations imposed at the inlets based on both the PODFS model that represents acoustic fluctuations and the PODFS model that represents turbulent fluctuations. In this case the pilot jet was seen to penetrate into the domain. This shows inlet turbulence must be included to ensure that the flow field and FTF is correctly captured and that the FTF of a burner is highly sensitive to the upstream turbulent field.

The large change in FTF phase between these two simulations shows that the FTF may be manipulated through changing the location of the flame inside the combustion chamber and opens a number of possibilities with respect to future designs. Future 
work will focus on whether the FTF can be derived from mean flow field.

The incompressible PODFS method has been tested against a fully compressible simulation at two frequencies. The method appears to offer similar levels of accuracy at a much reduced computational cost. This also shows that the mechanisms of heat release fluctuations are convective in nature and direct interactions between acoustic waves and the flame are of secondary importance in industrial lean-burn systems.

POD has been used to show that the downstream flow field of the simulation with the coring pilot penetration contains helical modes that are projected onto the fuel spray. Because of the higher momentum of the fuel spray this results in these helical modes penetrating deeper into the domain than in the case of gaseous fuelled combustors. This suggests that heat release fluctuations due to helical modes may be more significant in real liquid fuelled jet engines that gaseous fuelled lab-scale burners.

\section{Acknowledgement}

The authors wish to the thank Max Staufer and Marco Zedda at Rolls-Royce for their support. This work was funded by the EPSRC (Engineering and Physical Sciences Research Council) using grants EP/L015943/1 and EP/M023893/1 together with funding from Rolls-Royce. Some calculations were performed on HPC-midlands, grant ref . EP/K000063/1 and on ARCHER through the UK Applied Aerodynamics Consortium Leadership Project e529.

\section{Declaration of interest}

The authors have received funding from the EPSRC and Rolls-Royce.

\section{References}

Anand, M., Eggels, R., Staufer, M., Zedda, M., \& Zhu, J. (2013). An advanced unstructuredgrid finite-volume design system for gas turbine combustion analysis. In Asme 2013 gas turbine india conference (pp. V001T03A003-V001T03A003).

Candel, S., Durox, D., Schuller, T., Bourgouin, J.-F., \& Moeck, J. P. (2014). Dynamics of swirling flames. Annual review of fluid mechanics, 46, 147-173.

Caraeni, M., Devaki, R., Aroni, M., Oswald, M., \& Caraeni, D. (2009). Efficient acoustic modal analysis for industrial cfd. In 47 th aiaa aerospace sciences meeting including the new horizons forum and aerospace exposition.

Chomaz, J.-M. (2005). Global instabilities in spatially developing flows: non-normality and nonlinearity. Annu. Rev. Fluid Mech., 37, 357-392.

Chong, L. T. W., Komarek, T., Kaess, R., Foller, S., \& Polifke, W. (2010). Identification of flame transfer functions from les of a premixed swirl burner. In Asme turbo expo 2010: Power for land, sea, and air (pp. 623-635).

Dianat, M., McGuirk, J. J., Fokeer, S., \& Spencer, A. (2014). Les of unsteady vortex aerodynamics in complex geometry gas-turbine fuel injectors.

Dowling, A. P., \& Stow, S. R. (2003). Acoustic analysis of gas turbine combustors. Journal of propulsion and power, 19(5), 751-764.

Dupuy, F., Gatti, M., Mirat, C., Gicquel, L., Nicoud, F., \& Schuller, T. (2020). Combining analytical models and les data to determine the transfer function from swirled premixed flames. Combustion and Flame, 217, 222-236. 
Hermanns, R. (2001). Chem1d, a one-dimensional laminar flame code. Report, Eindhoven University of Technology.

Juniper, M. P. (2012). Absolute and convective instability in gas turbine fuel injectors. In Asme turbo expo, copenhagen, denmark, 11-15 june 2012 (p. GT2012-68253).

Keller, J., Gebretsadik, M., Habisreuther, P., Turrini, F., Zarzalis, N., \& Trimis, D. (2015). Numerical and experimental investigation on droplet dynamics and dispersion of a jet engine injector. International Journal of Multiphase Flow, 75, 144-162.

Kirthy, S. K., Hemchandra, S., Hong, S., Shanbhogue, S., \& Ghoniem, A. F. (2016). Role of shear layer instability in driving pressure oscillations in a backward facing step combustor. In Asme turbo expo 2016: Turbomachinery technical conference and exposition (pp. V04BT04A010-V04BT04A010).

Klein, M., Sadiki, A., \& Janicka, J. (2003). A digital filter based generation of inflow data for spatially developing direct numerical or large eddy simulations. Journal of computational Physics, $186(2), 652-665$.

Kolmogorov, A. (1941). The local structure of isotropic turbulence in an incompressible viscous fluid. In Dokl. akad. nauk sssr (Vol. 30, pp. 301-305).

Lambourne, N. (1962). The bursting of leading-edge vortices-some observations and discussion of the phenomenon. ARCR \& M., 3282, 1-36.

Langella, I., Heinze, J., Behrendt, T., Voigt, L., Swaminathan, N., \& Zedda, M. (2020). Turbulent flame shape switching at conditions relevant for gas turbines. Journal of Engineering for Gas Turbines and Power, 142(1).

Lefebvre, A., \& Chin, J. (1983). Steady-state evaporation characteristics of hydrocarbon fuel drops. AIAA journal, $21(10), 1437-1443$.

Menter, F., Kuntz, M., \& Langtry, R. (2003). Ten years of industrial experience with the sst turbulence model. Turbulence, heat and mass transfer, 4(1), 625-632.

Merk, M., Gaudron, R., Silva, C., Gatti, M., Mirat, C., Schuller, T., \& Polifke, W. (2019). Prediction of combustion noise of an enclosed flame by simultaneous identification of noise source and flame dynamics. Proceedings of the Combustion Institute, 37(4), 5263-5270.

Moeng, C.-H., \& Wyngaard, J. C. (1988). Spectral analysis of large-eddy simulations of the convective boundary layer. Journal of the Atmospheric Sciences, 45(23), 3573-3587.

Nehse, M., Warnat, J., \& Chevalier, C. (1996). Kinetic modeling of the oxidation of large aliphatic hydrocarbons. In Symposium (international) on combustion (Vol. 26, pp. 773$780)$.

Nicoud, F., Benoit, L., Sensiau, C., \& Poinsot, T. (2007). Acoustic modes in combustors with complex impedances and multidimensional active flames. AIAA journal, 45(2), 426-441.

Noiray, N., Durox, D., Schuller, T., \& Candel, S. (2008). A unified framework for nonlinear combustion instability analysis based on the flame describing function. Journal of Fluid Mechanics, 615, 139-167.

Oberleithner, K., Sieber, M., Nayeri, C., Paschereit, C., Petz, C., Hege, H.-C., .. Wygnanski, I. (2011). Three-dimensional coherent structures in a swirling jet undergoing vortex breakdown: stability analysis and empirical mode construction. Journal of Fluid Mechanics, 679, 383-414.

Palies, P., Schuller, T., Durox, D., \& Candel, S. (2011). Modeling of premixed swirling flames transfer functions. Proceedings of the combustion institute, 33(2), 2967-2974.

Palies, P., Schuller, T., Durox, D., Gicquel, L., \& Candel, S. (2011). Acoustically perturbed turbulent premixed swirling flames. Physics of Fluids, 23(3), 037101.

Polifke, W. (2015). Six lectures on thermodynamic combustion instability. 21st CISM-IUTAM international summer school on measurement, analysis and passive control of thermoacoustic oscillations.

Polifke, W., \& Lawn, C. (2007). On the low-frequency limit of flame transfer functions. Combustion and flame, 151(3), 437-451.

Pope, S. B. (2001). Turbulent flows. IOP Publishing.

Renaud, A., Ducruix, S., \& Zimmer, L. (2019). Experimental study of the precessing vortex core impact on the liquid fuel spray in a gas turbine model combustor. Journal of 
Engineering for Gas Turbines and Power, 141(11).

Robin, V., Mura, A., Champion, M., \& Plion, P. (2006). A multi-dirac presumed pdf model for turbulent reactive flows with variable equivalence ratio. Combustion science and technology, 178(10-11), 1843-1870.

Schuller, T., Durox, D., \& Candel, S. (2003). A unified model for the prediction of laminar flame transfer functions: comparisons between conical and v-flame dynamics. Combustion and Flame, 134(1), 21-34.

Sirovich, L. (1987). Turbulence and the dynamics of coherent structures. i. coherent structures. Quarterly of applied mathematics, 45(3), 561-571.

Smagorinsky, J. (1963). General circulation experiments with the primitive equations: I. the basic experiment. Monthly weather review, 91(3), 99-164.

Steinberg, A. M., Boxx, I., Stöhr, M., Meier, W., \& Carter, C. D. (2012). Effects of flow structure dynamics on thermoacoustic instabilities in swirl-stabilized combustion. AIAA journal, 50(4), 952-967.

$\mathrm{Su}$, J., Garmory, A., \& Carrotte, J. (2015). The aerodynamic response of fuel injector passages to incident acoustic waves. In Asme 2015 turbine technical conference and exposition (p. GT2015-43248).

Terhaar, S., Ćosić, B., Paschereit, C. O., \& Oberleithner, K. (2016). Suppression and excitation of the precessing vortex core by acoustic velocity fluctuations: An experimental and analytical study. Combustion and Flame, 172, 234-251.

Thumuluru, S. K., \& Lieuwen, T. (2009). Characterization of acoustically forced swirl flame dynamics. Proceedings of the Combustion Institute, 32 (2), 2893-2900.

Treleaven, N. C. W., Garmory, A., \& Page, G. J. (2019). The effect of sauter mean diameter fluctuations on the heat release rate in a lean-burn aero-engine combustor. In Asme 2019 turbine technical conference and exposition (p. GT2019-90321).

Treleaven, N. C. W., Garmory, A., \& Page, G. J. (2020). A numerical study on the effects of acoustic forcing on fuel spray dynamics in gas turbines. In Accepted to: Asme 2020 turbine technical conference and exposition (p. GT2020-14238).

Treleaven, N. C. W., Juniper, M., Su, J., Garmory, A., \& Page, G. J. (2018). The identification and prediction of helical modes induced by a multi-passage swirl stabilised lean burn aeroengine fuel injector under steady state and acoustically forced conditions. In Asme 2018 turbine technical conference and exposition (p. GT2018-75552).

Treleaven, N. C. W., Staufer, M., Spencer, A., Garmory, A., \& Page, G. J. (2020). Application of the podfs method to inlet turbulence generated using the digital filter technique. Accepted to: Journal of Computational Physics.

Treleaven, N. C. W., Su, J., Garmory, A., \& Page, G. J. (2017). The response to incident acoustic waves of the flow field produced by a multi-passage lean-burn aero-engine fuel injector. In Asme 2017 turbine technical conference and exposition (p. GT2017-64527).

Treleaven, N. C. W., Su, J., Garmory, A., \& Page, G. J. (2019). An efficient method to reproduce the effects of acoustic forcing on gas turbine fuel injectors in incompressible simulations. Flow, Turbulence and Combustion, 1-21.

Vicquelin, R., Fiorina, B., Payet, S., Darabiha, N., \& Gicquel, O. (2011). Coupling tabulated chemistry with compressible cfd solvers. Proceedings of the Combustion Institute, 33(1), $1481-1488$.

Vold, H., Crowley, J., \& Rocklin, G. T. (1984). New ways of estimating frequency response functions. Sound \& Vibration, 18(11), 34-38.

Williams, M. A., Carrotte, J. F., Moran, A. J., \& Walker, A. D. (2018). Impact of upstream boundary conditions on fuel injector performance in a low trl reacting flow experimental facility. In Asme turbo expo 2018: Turbomachinery technical conference and exposition.

\section{Tables}


Table 1. Approximate cost of the compressible and incompressible methods in CPUh.

\begin{tabular}{lccc}
\hline Method & Cells & Timesteps & CPUh \\
\hline Compressible & & & $\mathbf{1 4 6 k}$ \\
$1 \times$ Reacting compressible & $15 \mathrm{M}$ & $100 \mathrm{k}$ & $146 \mathrm{k}$ \\
\hline PODFS & & & $\mathbf{4 4 k}$ \\
$1 \times$ Non-reacting compressible & $5 \mathrm{M}$ & $100 \mathrm{k}$ & $27 \mathrm{k}$ \\
$1 \times$ Reacting incompressible & $6 \mathrm{M}$ & $40 \mathrm{k}$ & $17 \mathrm{k}$ \\
\hline
\end{tabular}

Table 2. The azimuthal wavenumbers $(m)$ for the velocity components of the first 10 POD modes for the reacting coring LES simulation together with the results from the compressible non-reacting auxiliary computations.

\begin{tabular}{lccc}
\hline Simulation & $\begin{array}{c}\text { Compressible } \\
\text { non-reacting } \\
\text { URANS }\end{array}$ & $\begin{array}{c}\text { Incompressible } \\
\text { non-reacting } \\
\text { LES }\end{array}$ & $\begin{array}{c}\text { Incompressible } \\
\text { reacting } \\
\text { LES }\end{array}$ \\
\hline Mode 1 & 2 & 2 & 2 \\
Mode 2 & 2 & 2 & 2 \\
Mode 3 & 0 & 1 & 1 \\
Mode 4 & 0 & 1 & 1 \\
Mode 5 & 0 & 3 & 0 \\
Mode 6 & 3 & 0 & 0 \\
Mode 7 & 0 & 3 & 0 \\
Mode 8 & 3 & 1 & 3 \\
Mode 9 & 3 & 1 & 3 \\
Mode 10 & 3 & 1 & 3 \\
\hline
\end{tabular}

Figures 


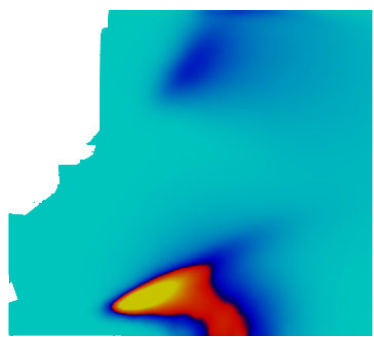

(a) Coring flow.

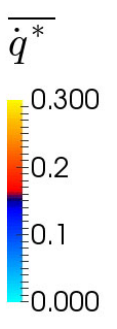

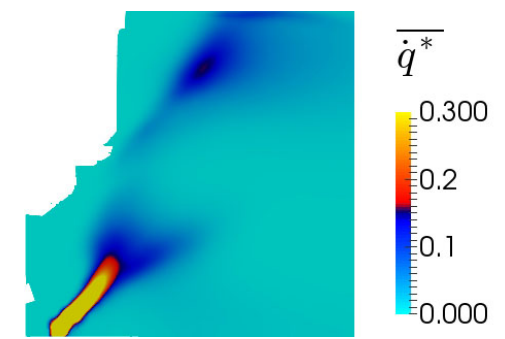

(b) Flipped flow.

Figure 1. The time and azimuthally averaged heat release rate from the coring and flipped simulations.

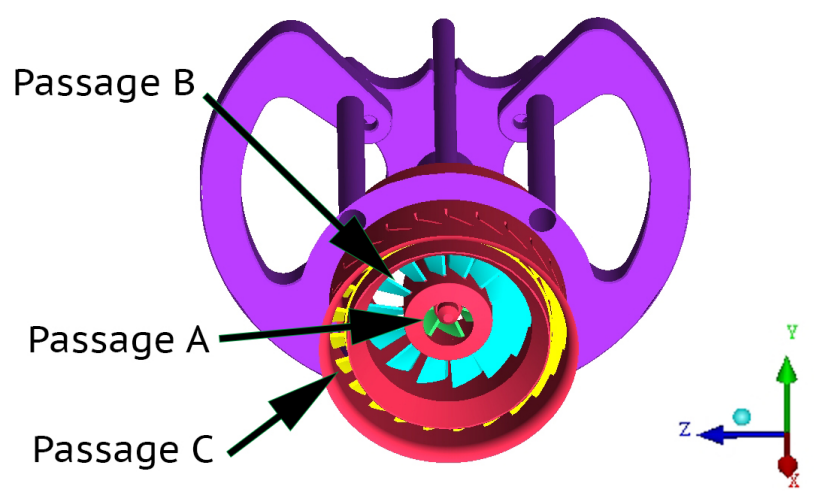

Figure 2. The full injector geometry used in the auxiliary compressible URANS simulation.

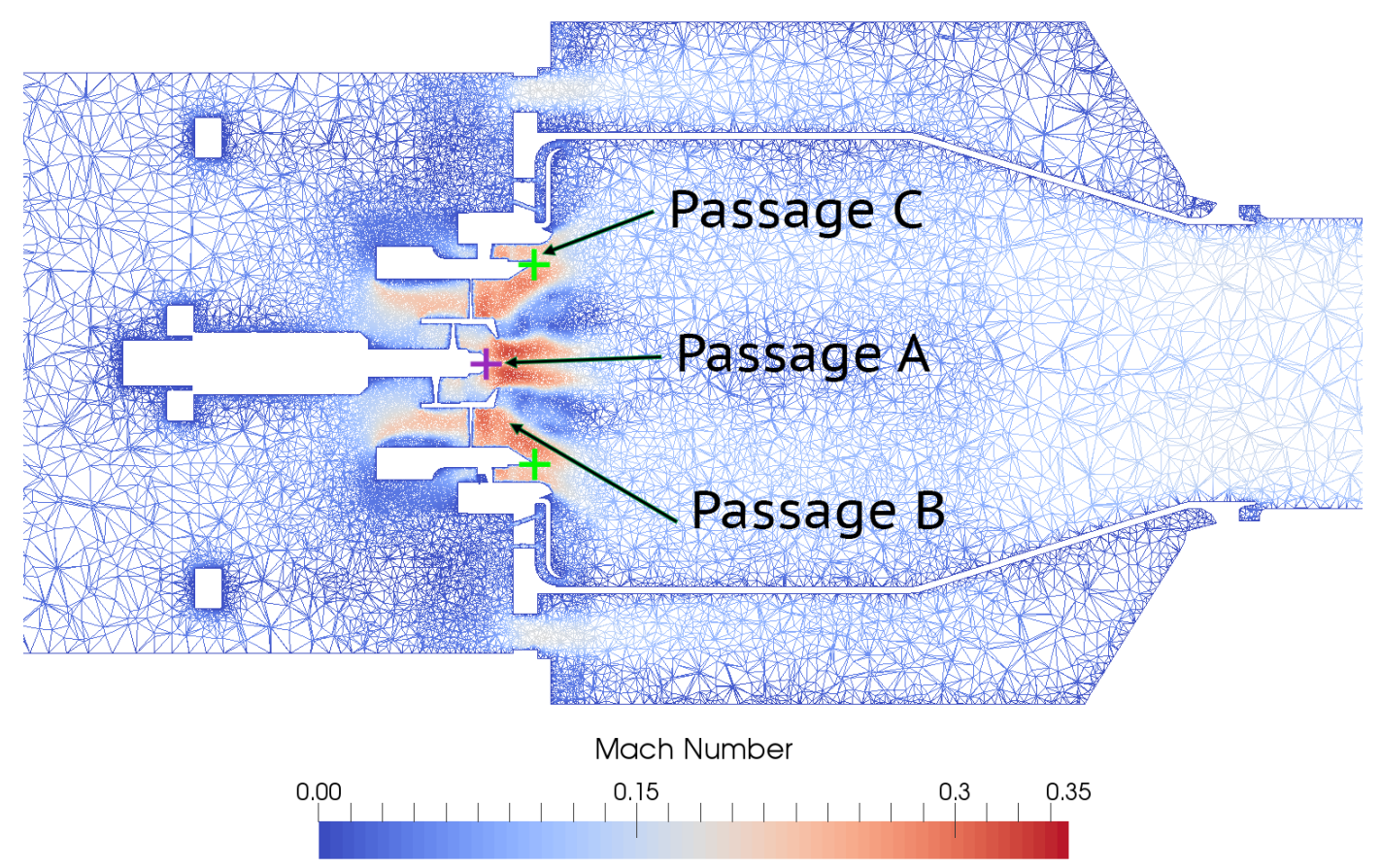

Figure 3. The mesh and Mach Number of the mean flow around the injector for the auxiliary compressible URANS simulation. 


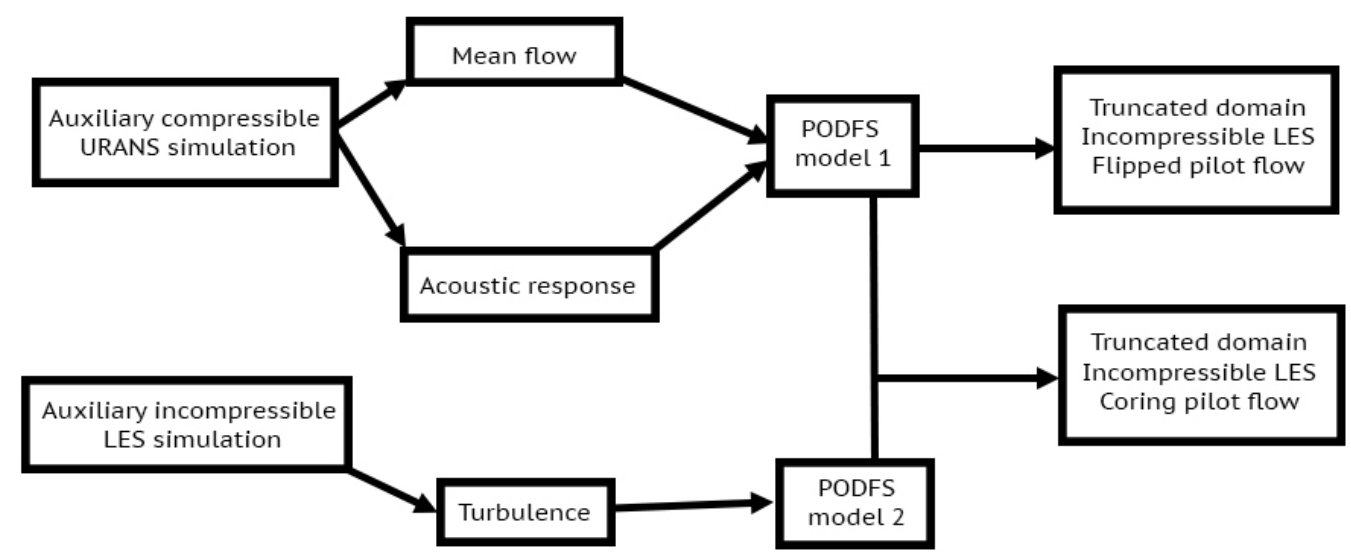

Figure 4. The workflow presented in this paper.

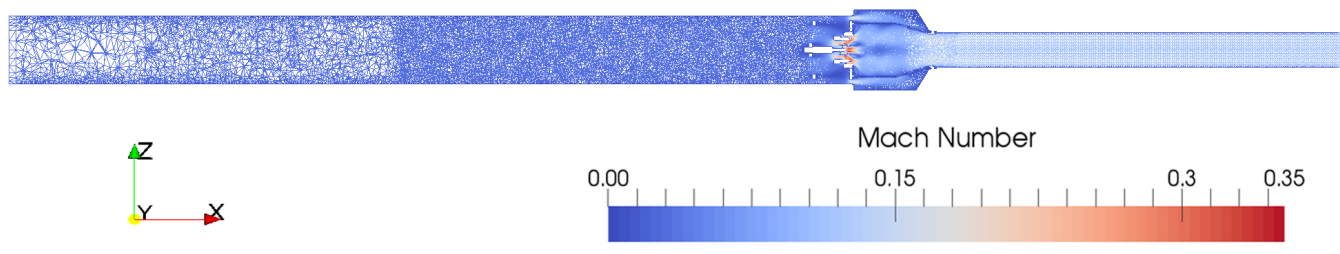

Figure 5. The mesh and Mach Number of the mean flow for the auxiliary compressible URANS simulation.

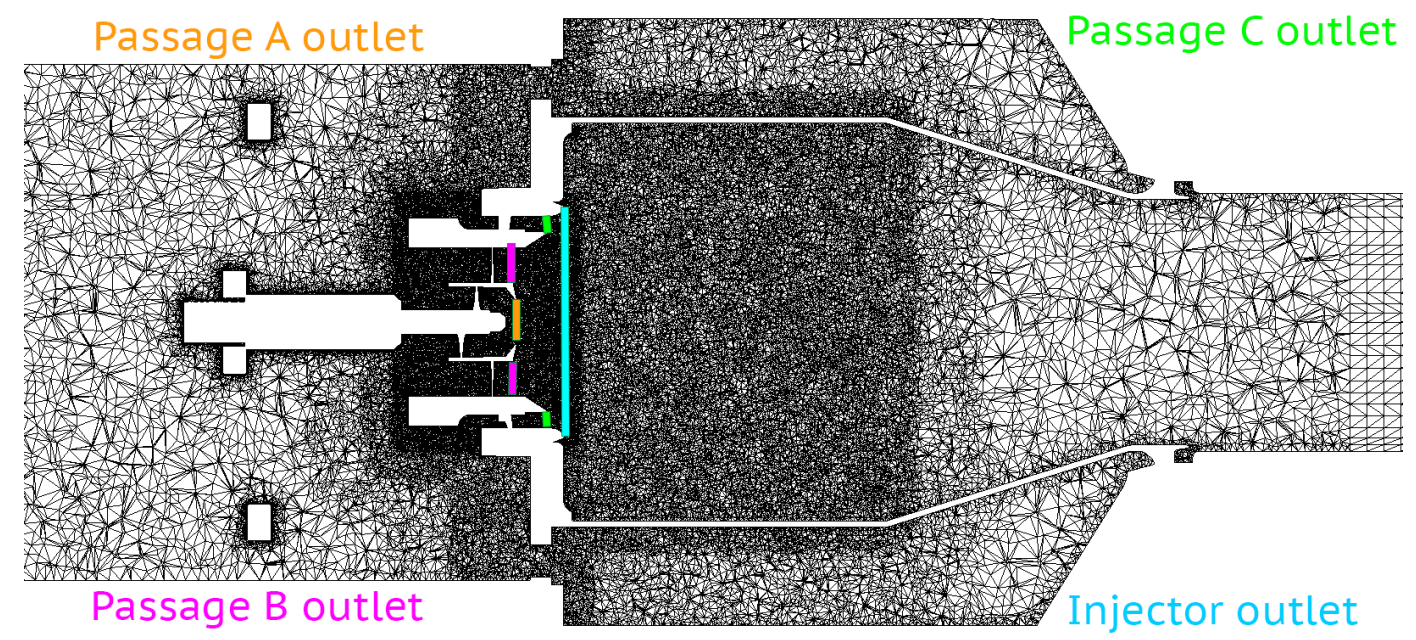

Figure 6. The centre section of the mesh used for the compressible reacting simulations. 


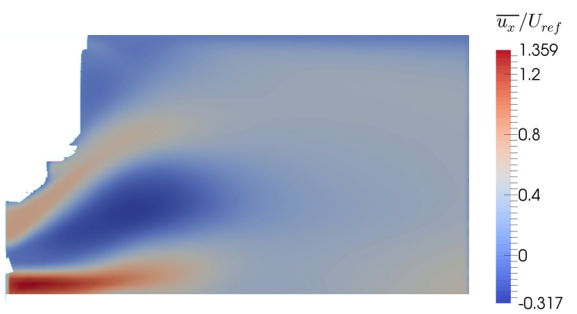

(a) Coring flow.

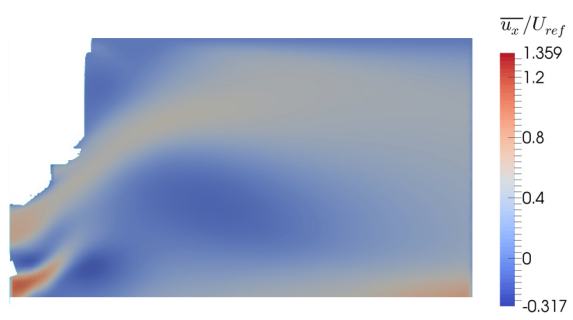

(b) Flipped flow.

Figure 7. The time and azimuthally averaged axial velocity field from the coring and flipped simulations.

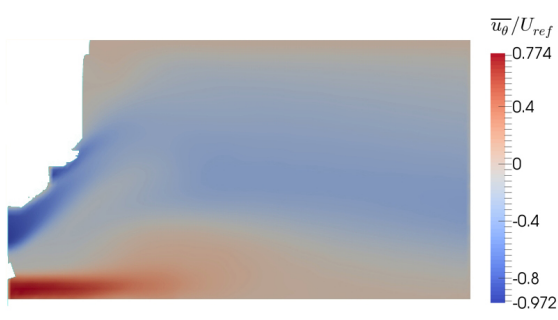

(a) Coring flow.

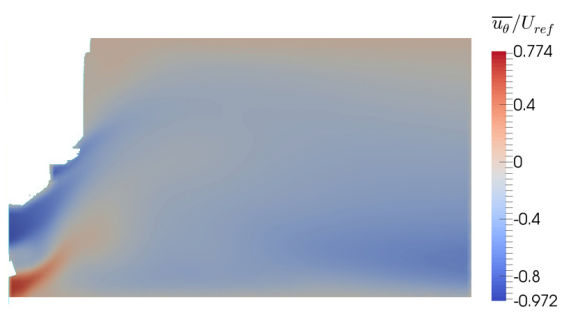

(b) Flipped flow.

Figure 8. The time and azimuthally averaged azimuthal velocity field from the coring and flipped simulations.

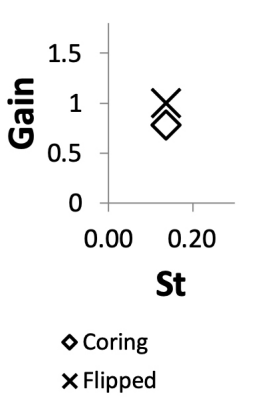

(a) Gain.

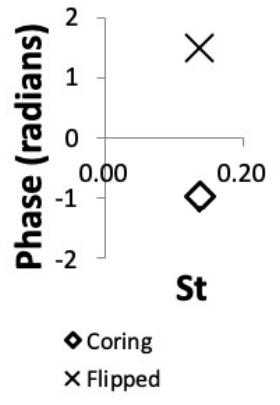

(b) Phase.

Figure 9. The calculated FTF from the coring and flipped simulations. 


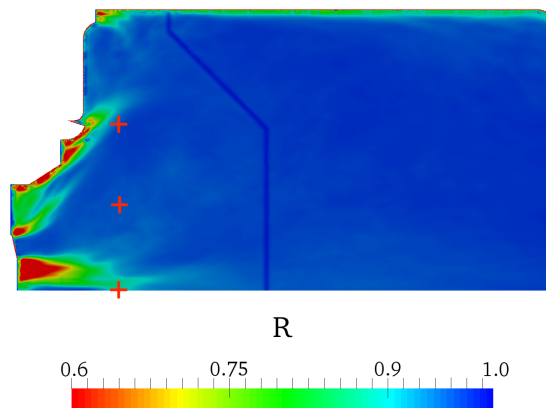

(a) The proportion of resolved turbulent kinetic energy.

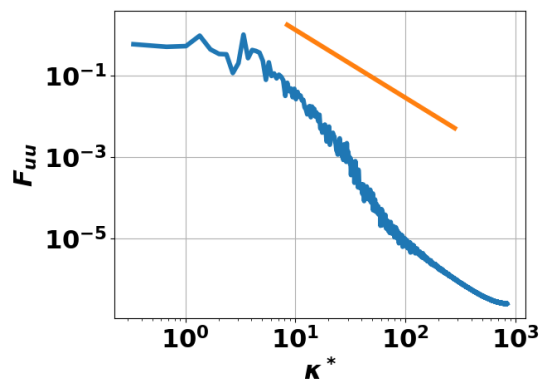

(c) The turbulent spectra at the middle point.

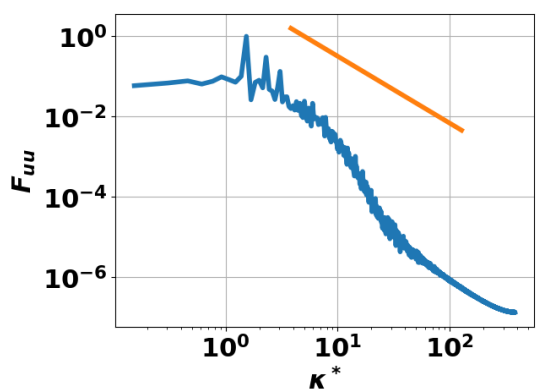

(b) The turbulent spectra at the outer point.

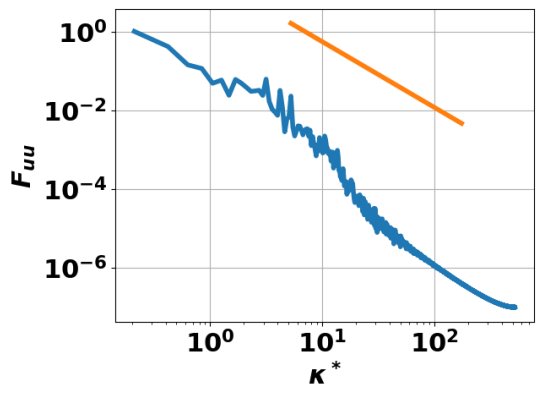

(d) The turbulent spectra at the bottom point.

Figure 10. The proportion of turbulent kinetic energy resolved in the LES simulation of the coring case and the one-dimensional turbulent spectra at the points marked with a red cross. The spectra are also shown with a line with a gradient of $-5 / 3$ in orange. $\kappa^{*}=\kappa D$ is the non-dimensional wavenumber.

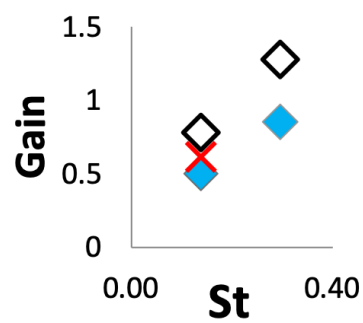

$\diamond$ Compressible

$\diamond$ Coring

$X$ Low resolution

(a) Gain.

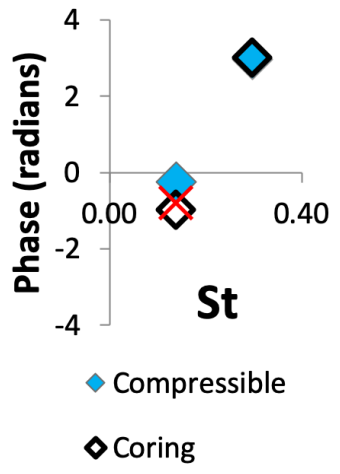

$X$ Low resolution

(b) Phase.

Figure 11. The calculated FTF from the coring high resolution incompressible simulations along with the low resolution and compressible cases. 


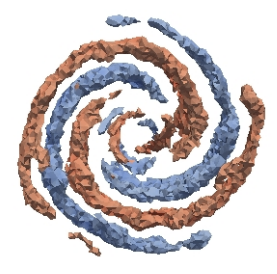

(a) Axial velocity.

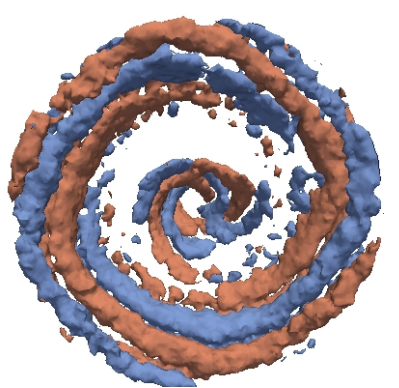

(c) Mixture fraction.

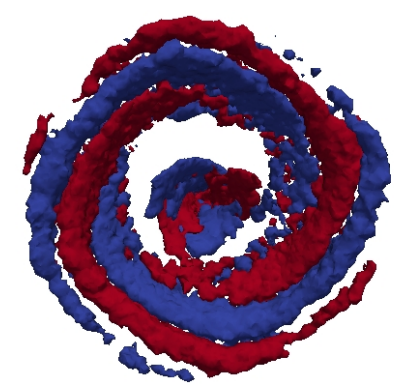

(b) Spray axial momentum.

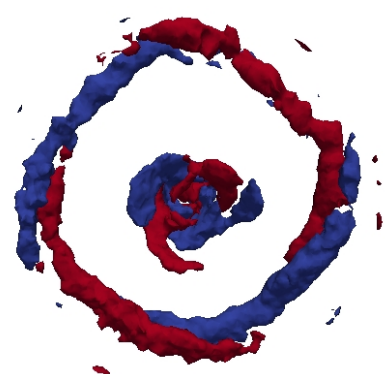

(d) Heat release rate.

Figure 12. Iso-surfaces of the 2 nd most energetic POD mode for the coring simulation forced at $S t=0.14$ as observed from downstream. Red symbolises a positive scalar value and blue a negative value. 